\title{
A biased probe analysis of potential well formation in an electron only, low beta Polywell magnetic field
}

\author{
Matthew Carr and Joe Khachan \\ Department of Plasma Physics, School of Physics A28, University of Sydney, \\ NSW 2006, Australia
}

(Dated: 18 March 2013)

Orbital limited motion theory has been applied to two biased probes in a low beta Polywell. The cases studied include electron injection, magnetic field scaling, Polywell bias scaling, and radial position profiles. Langmuir's original orbital limited motion results for a monoenergetic electron beam are shown to be in excellent agreement for electron injection into the Polywell. A distribution function is proposed for the electron plasma characteristics in the centre of the magnetic null and confirmed with experimental results. A translational stage was used to measure the radial plasma potential profile. In other experiments two probes were used to simultaneously measure the profiles in both the null and a position halfway along a corner cusp. The results confirm a radial potential well created by electron trapping in the device. In addition, we present preliminary results of the potential well scaling with the magnetic field, Polywell bias voltage, and the injected beam current. The electron population was found to maintain non-equilibrium in all cases studied. 


\section{THE POLYWELL CONCEPT}

The Polywell is a hybrid fusion device based on concepts from inertial electrostatic confinement (IEC) ${ }^{1-3,5-8}$ and cusped magnetic confinement fusion ${ }^{9,10}$. The Polywell ${ }^{11-14,16-18}$ uses a cusped magnetic field to trap electrons that create a negative spherical potential well for trapping positive ions. Ions created at the edge of the device are accelerated to fusion relevant energies and confined by the potential well. The magnetic field required for trapping energetic electrons is much less than the corresponding B needed for energetic ions because of the much larger charge to mass ratio, $q / m$, of the electrons. Hence in principle the problem of trapping energetic ions is replaced by the problem of energy loss by electron transport across field lines. The use of this concept is aimed at solving a key limitation discovered in gridded IEC systems through the formation of a virtual cathode ${ }^{4,5}$.

The unique magnetic field configuration of the Polywell is created by pairs of opposing current loops, each creating a cusp about the origin. In the cube configuration each pair of loops is centred on a Cartesian axis such that each loop is on a face of the cube. The opposing contributions from each current loop cancel out in the centre of the device creating a magnetic null point. The resulting field acts like a magnetic well and a proportion of the electron population is confined by the magnetic mirror effect ${ }^{19}$. The magnetic field structure of the Polywell is shown in Figure 1 overlaid with the magnetic field lines. By using a virtual cathode there is no longer a loss surface embedded within the plasma. The outer grid that contains the magnetic field coils is effectively isolated by the magnetic field it creates ${ }^{14}$. Further improvement to confinement may occur at high densities where diamagnetic effects are expected to push out the fieldlines into a Wiffleball configuration ${ }^{14}$. In this mode, normal mirror confinement would be replaced by ballistic "Wiffleball" confinement leading to reduced energy loss.

A biased probe diagnostic enables a number of parameters to be measured that are crucial to a Polywell's performance. A single ended Langmuir probe provides a local measurement of the plasma potential at the probe position and hence can be used to directly map the spatial potential profile within the device. Characterising the potential well structure is crucial to any experimental study of the Polywell because the formation of a deep virtual cathode is integral to the operation of a Polywell ${ }^{14}$. In addition, a biased probe allows the determination

of the Electron Energy Distribution Function (EEDF) and electron density, $n_{e}$. Understand- 
ing the EEDF is critical for measuring the relative energy components for monoenergetic (beam like) and thermal motion. In an ideal Polywell electrons would be monoenergetic to avoid large energy losses through energy upscattering. However in any real plasma system we expect thermalisation to occur, hence we also want to measure the temperature of the electrons, $T_{e}$. Rosenberg and Krall have theoretically proposed that the Polywell should be able to support a non-maxwellian plasma on moderately long time $\operatorname{scales}^{20}$. Measuring the ratio of the monoenergetic and thermal components of the electron energy distribution enables the degree of thermalisation in the plasma to be determined and is important for determining whether a monoenergetic electron population can be sustained ${ }^{20}$.

A biased cylindrical Langmuir probe can be used to measure all of these parameters but is not usually applied to experiments with a high (keV) electron energy or with a magnetic field. At the high bias voltages required for measuring keV electrons the probe can create its own discharge corrupting the results. However the underlying confinement properties scale with magnetic field strength and energy, and thus can be studied with low energy electrons without fundamentally changing the physics.

In magnetic fields the analysis of biased probes can become extremely complicated and sometimes intractable. In general, there is no exact theory about the interpretation of the current versus voltage $\mathrm{I}(\mathrm{V})$ trace of a single ended Langmuir probe in a magnetic field. However, the Polywell has a magnetic null in the centre of the device which can be used to exactly characterise the electron parameters. The magnetic field varies with $r^{3}$ radially outwards from the null, and hence we can use a second probe to measure the gradual change in the $\mathrm{I}(\mathrm{V})$ traces, assuming the deviation due to the presence of the magnetic field is initially small. Eventually one expects the I(V) trace to slowly diverge from the theoretically expected curve, producing increasingly larger errors.

The only previous experiment to measure the plasma potential of the Polywell was performed by Krall using a capacitive probe $^{13}$. The capacitive technique is expected to be superior in larger fusion relevant Polywells where large electron energies and higher electron densities may make the single biased probe method unusable. However, when the physics is scaled down to a comparatively low electron energy and magnetic field regime the biased probe method is advantageous because of its measurement accuracy and scope of parameters measured.

The principle aim of this work is to apply two biased probes to measure the radial poten- 
tial well profile as a function of other parameters such as magnetic field, injection current and injection energy. We will also present preliminary measurements of the way the potential well scales with the Polywell bias voltage and magnetic field strength. Furthermore, we will comment on the relationships between other parameters measured such as the electron density, mean energy and thermal energy component.

\section{EXPERIMENTAL SETUP}

The Polywell was constructed from 6 coils each with 15 turns of enamelled copper wire. These coils were mounted into coil formers that were Aluminium torus shells made using a metal spinning procedure. The coils were driven from a pulsed current power supply that consisted of a $7.5 \mathrm{mF}$ capacitor bank, which could be charged to a maximum voltage of $450 \mathrm{~V}$. The capacitors were discharged into the Polywell coils through a triggered silicon controlled rectifier (SCR). The peak current attainable varied between $50 \mathrm{~A}$ and $2.5 \mathrm{kA}$ by changing the voltage applied to the capacitors. A second power supply utilised two $12 \mathrm{~V}$ car batteries in series, with an SCR to provide a pulsed constant current source with current range 0 to $50 \mathrm{~A}$.

The Polywell was mounted on a cross-beam in the centre of a cylindrical vacuum chamber, see Figure 2. The electron guns were 6 light bulb filaments, each mounted so as to be centred on a coil face at a distance of $2 \mathrm{~cm}$ from the outside plane of a coil face. The toroidal Aluminium shells of the coils were biased with a positive potential in order to extract the electrons from the filaments and to accelerate them into the interior of the device. Although it might be advantageous in future to employ a more sophisticated 3 terminal configuration, this design was chosen to mimic Bussard's original WB6 configuration ${ }^{14}$. Despite the fact that the experiment included a mass flow system for injection of hydrogen gas, all results reported in this paper were carried out in moderate vacuum conditions with a pressure $<10^{-5}$ Torr. For simplicity, our initial experiments were aimed at determining the electron behaviour in the absence of any background gas.

Two cylindrical Langmuir probes were constructed from tungsten wire with a radius $r_{p}=50 \mu \mathrm{m}$ and mounted into ceramic tubing with a $0.8 \mathrm{~mm}$ outer diameter. The part of the tungsten wire that extends into the plasma is the probe length, $l_{p}=5 \mathrm{~mm}$. Probe A was mounted onto a translation stage with a total travel distance of $5.5 \mathrm{~cm}$ and moved in 3 
$\mathrm{mm}$ increments. It was positioned along a coil axis so that the extremities of translation are the geometric centre of the Polywell and a location just outside the Polywell coils. Probe A was at an angle of $8^{\circ}$ to the coil axis so that it did not block the filament that was the source of electrons to this face of the Polywell. An additional reason for having a small angle is so that the probe crosses more magnetic field lines. Electron transport along magnetic field lines is much faster than cross field transport. This is expected to accelerate space potential build up across field lines and would result in a larger potential difference than would occur along a point cusp. Probe B is permanently mounted in alignment with a corner cusp and located halfway along the Polywell radius.

Both probes were driven from a PA241 high voltage op-amp. An Arduino Mega 2560 was used as a configurable function generator for controlling parameters such as sweep time, pulse height and shape, etc. Both probes were swept from $-140 \mathrm{~V}$ to $+140 \mathrm{~V}$ in a sweep time of $100 \mu \mathrm{s}$. Current signals were measured directly on an oscilloscope. Current resolution of up to $1 \mu \mathrm{A}$ was possible. The driving circuit is shown in Figure 3, which also features a two pole Bessel filter for noise suppression.

\section{ORBITAL LIMITED MOTION LANGMUIR PROBE THEORY}

The most appropriate type of theory to use is an orbital limited motion theory which was originally described by Langmuir and Mott-Smith ${ }^{23}$. Based on the emission current of our filaments, we expect to observe densities between $10^{8}-10^{9} \mathrm{~cm}^{-3}$ at energies that give us debye lengths, $\lambda_{d}$, ranging from $2 \mathrm{~cm}$ down to $1 \mathrm{~mm}$. Hence it is assumed that the device radius $R_{\text {device }}>\lambda_{d} \gg r_{p}$ at all times. In the low density limits we expect to have inaccuracies when the $\lambda_{d}$ is almost as large as the device. However, even before this limit is reached, the characteristic curve will start to deviate from the theory as $\lambda_{d} \approx l_{p}$ and the infinite cylinder approximation is no longer applicable. It was assumed that the sheath around a probe has a sharp edge boundary. The potential at this boundary was considered to be the plasma potential.

We also assumed that the electron plasma is collisionless such that the electron motion in the sheath around the probe can be described by free orbits. Because $r_{p} \ll l_{p}$ we can assume the sheath is cylindrically symmetric and probe end effects can be ignored. Consequently, the conditions that determine whether an electron will reach the collector for a given voltage 
depend only on the space potential and initial electron velocity at the sheath edge. Therefore the total I(V) curve for electron current collected at the probe is determined by summing the contributions from the electron distribution function with the appropriate initial conditions. The resulting equation is only a function of the potential across the sheath and the sheath radius. For the calculation to be exact it is also necessary to solve Poisson's equation for the space charge in the sheath. This approach has been carried out on a number of occasions ${ }^{26}$ however we will assume that the problem is independent of sheath size and focus on orbital limited motion.

In cylindrical symmetry the current is calculated in a $2 \mathrm{D}$ plane intersecting the probe, giving current per unit probe length, $I / l$. The velocity co-ordinates $u$ and $v$ are defined as the radial and tangential velocity components, respectively, of an electron arriving at the probe sheath. The velocity distribution function, $f(u, v)$ is normalised such that

$$
n_{e} f(u, v) d u d v
$$

gives the number of electrons per unit volume with velocity components in $d u$ and $d v$. Therefore, the number of electrons in unit time that arrive at the sheath edge with velocities in $d u$ and $d v$ are

$$
2 \pi a n_{e} u f(u, v) d u d v
$$

where $a$ is the sheath radius and $n_{e}$ is the electron density. In its current form, this equation is challenging to use because the sheath radius is an unknown. However, Langmuir has shown ${ }^{23}$ that in the limit of an infinite sheath, equation 2 approaches a limiting form. By taking the limit of a large sheath in equation 2 , multiplying by the charge $q$, length $l_{p}$, and integrating over the limits of velocity for electrons that can reach the probe, Langmuir obtained the general form for the current collected by a cylindrical probe ${ }^{23}$.

$$
I(V)=4 \pi r_{p} l_{p} n_{e} q \int_{0, v_{0}}^{\infty} u \sqrt{u^{2}+\frac{2 q\left(V-V_{p}\right)}{m_{e}}} f(u, 0) d u .
$$

Here $V$ is the probe bias voltage. The lower boundary of velocity integration is split based on whether the probe is attractive or repulsive with respect to the local plasma potential, $V_{p}$. When the probe is attractive, $V>V_{p}$, the lower bound is 0 since all electrons have enough energy to potentially reach the probe surface. However, when the probe is negatively biased, $V<V_{p}$, electrons must have at least some critical minimum energy in order to overcome the 
repulsive field from the probe and reach the probe surface. This critical minimum energy is determined by the voltage difference between the probe and the plasma, and can be expressed as a minimum velocity $v_{0}$, where

$$
v_{0}=\sqrt{\frac{2 q\left(V-V_{p}\right)}{m_{e}}} .
$$

This equation was used by Langmuir and Mott-Smith ${ }^{23}$ to derive the ubiquitous maxwellian $I_{m}(V)$ characteristic as well as the $I_{b}(V)$ for a one dimensional beam. However, these results are only applicable in plasmas with the appropriate distribution functions and become meaningless in plasmas where extra complicating factors such as a magnetic field can significantly alter the EEDF. To make the process of analysing the probe data tractable it is important to build up information about the EEDF at each phase of progressively more complex plasma conditions.

In the case of the Polywell this means at first understanding and confirming the characteristics of the electrons being injected into the Polywell. This information can be used to approximate the EEDF in the centre of the well when a relatively weak magnetic field is applied. And finally we can build on these results to make measurements of electrons in a moderate magnetic field. At each stage the base $I(V)$ equation (3) for the biased probe response can be directly modified for the specific plasma conditions anticipated. As long as the expected distribution function is known we can integrate this function numerically and compare it with the data, and use it to fit plasma parameters to the measured $I(V)$ data.

\section{MEASUREMENTS OF ELECTRON INJECTION}

The electron source (the heated filament) was at ground potential. Electrons were extracted and accelerated by the electrostatic potential placed on the metal casing of the Polywell field coils, thus accelerating the electrons in a direction that is normal to the plane of the coils. As a result, the energy distribution function of the electron beam was expected to be monoenergetic. However, one can also expect some spreading in this energy distribution due to interactions of the electrons with the local space charge established by the beam.

We have assumed that the magnetic field lines at the centre plane of a coil, and therefore approximately parallel to the direction of motion of the beam, would not change the energy 
distribution greatly. As a result, we approximated the electron distribution function as that given by Langmuir ${ }^{23}$ for a beam of electrons with a Maxwellian energy component given by

$$
\begin{array}{r}
f_{b}(u, v, \theta)=\frac{m_{e}}{2 \pi k T} \exp \left\{-\frac{m_{e}}{2 k T}\left(u^{2}+v^{2}+u_{d}^{2}\right.\right. \\
\left.\left.-2 u_{d}(u \cos \theta+v \sin \theta)\right)\right\},
\end{array}
$$

where $u_{d}$ is the drift velocity of the beam. The coordinate $\theta$ is the angle between the beam axis along $u_{d}$ and the radial velocity coordinate, $u$, with respect to the sheath. In the limit of a large sheath, the sheath can be approximated as being circular. Hence the probe current is found by using $f_{b}(u, v, \theta)$ in Equation 3 and integrating around the sheath circumference ${ }^{23}$,

$$
\begin{gathered}
I_{b}(V)=2 \pi r_{p} l_{p} n_{e} q \\
\int_{0}^{2 \pi} \int_{0, v_{0}}^{\infty} u \sqrt{u^{2}+\frac{2 q\left(V-V_{p}\right)}{m_{e}}} f_{b}(u, 0, \theta) d u d \theta .
\end{gathered}
$$

When the probe is at the plasma potential $V_{p}$, the collected probe current can be approximated as

$$
I_{0}=\frac{1}{4} A_{p} n_{e} q \bar{c}
$$

where $A_{p}$ is the probe surface area and $\bar{c}$ is the mean speed of the electron distribution ${ }^{27}$. In the case of the beam distribution $f_{b}, \bar{c}=u_{d}$.

Equation 6 cannot be evaluated analytically. Heatley ${ }^{24}$ has found an exact series solution but it converges very slowly when the drift velocity is large compared with the thermal velocity. Since this scenario is expected in our situation, we have obtained solutions through numerical evaluation of equation 6 .

The measured $\mathrm{I}(\mathrm{V})$ data was filtered using a moving window average combined with a Svatsky and Golay filter ${ }^{21,22}$. The data was fitted using the standard non-linear fitting tools available in Mathematica. The only constraint applied to the fitted model was that $V_{p}<V_{\text {bias }}$, since it is not possible for the plasma potential $V_{p}$ to be more positive than the Polywell bias potential $V_{\text {bias }}$ in an electron only plasma.

A sample fit is shown with the fitted parameters in Figure 4. All fit parameters are reported in their voltage equivalent energies to make for easy comparisons with other experimental parameters. If electrons are accelerated from ground through to $V_{p}$ then they are expected to have a monoenergetic drift energy, $V_{d}$, close to but less than $V_{p}$, which is observed. Since the electron density and beam transit times are small, such that the electron 
collision frequency is negligible, we also expect the thermal energy component, $V_{t h}$, to be much less than the drift energy. The fitted parameters are in the order $V_{\text {bias }}>V_{p}>V_{d} \gg V_{t h}$, and support the hypothesis that this experimental setup results in a beam of monoenergetic electrons with a very small thermal energy.

A further justification for the use of the drift maxwellian distribution over the most commonly occurring normal maxwellian distribution, $f_{m}$, is now given by examining the differences between both $I(V)$ characteristics. The characteristic $I(V)$ for $f_{m}, I_{m}(V)$, consists of two regions, one on either side of the plasma potential. The $I_{m}(V)$ is $\propto e^{V}$ when the probe is repulsive, and $\propto \sqrt{V}$ when attractive. The plasma potential can then be found by plotting the double derivative, $d^{2} I / d V^{2}$, and finding the zero crossing point which is equal to $V_{p}$. This is because the two halves of the $I_{m}(V)$ around $V_{p}$ have opposite curvature. By following this standard procedure we can show that our data differs substantially from the normal maxwellian characteristic, $I_{m}(V)$, and hence a poor fit was obtained. There is significant deviation in the fit residuals around the $V_{p}$ given by $d^{2} I / d V^{2}=0$. This procedure proved that the standard maxwellian distribution is not suitable, but does not prove that the drifting maxwellian is the best choice.

Because of the complex geometry this is likely to be an oversimplification and other distributions could arise. In some cases, in particular cases with low extraction voltage, an appropriate fit to either distribution could not be obtained. Consequently, one must assume there might be another more appropriate distribution. Consider that at lower extraction voltages space charge effects can significantly alter the distribution shape. A 1D vacuum diode approximation to this geometry suggests space charge effects may occur in the lower extraction voltage range of this experiment.

\section{WITH B AND WITHOUT B TEST CASES}

By building on the information learned about the EEDF of extracted electrons in the previous section, we can now begin to analyse how a biased probe will respond when placed in the magnetic null at the centre of the device. When the magnetic field is turned off, $B_{\text {peak }}=0$, we can use the same drifting maxwellian as that given in Eqn. 6. However, when the magnetic field is turned on, one needs to take into account the effects of the magnetic field structure on the EEDF in the null. As discussed in a previous paper ${ }^{17}$, the magnetic 
null region causes an electron beam to become defocused and undergo ballistic collisions around the adiabatic flux surface. This ballistic scattering effect leads to an electron energy distribution that is isotropic and predominantly monoenergetic with a narrow spread of electron energies due to thermalisation. These assumptions are expected to be valid only in the absence of space charge effects for a low density electron plasma, which were the experimental conditions reported in this paper. The resulting distribution could be described as a maxwellian with an isotropic mean offset energy, $V_{\mu}$. The distribution we propose is

$$
f_{\mu}\left(v_{r}\right)=e^{-\frac{m\left(v_{r}-v_{\mu}\right)^{2}}{2 \sigma^{2}}}
$$

where $v_{r}$ is the velocity component along the radial coordinate in spherical coordinates, $v_{\mu}$ is the velocity equivalent to the offset energy $V_{\mu}, \sigma$ is the standard deviation of the velocities and is related to the thermal energy $V_{t h}$, through $\sigma^{2}=q V_{t h}=k T_{e}$. Here it is possible to define an electron temperature $T_{e}$ although it is not strictly true in the conventional sense. This distribution function is isotropic because there is no dependence on the angular velocity coordinates $v_{\theta}$ or $v_{\phi}$. A plot of $f_{\mu}\left(v_{r}\right)$ is given in Fig. 5 .

Two limits are imposed on the proposed velocity distribution as a check to its validity. First, in the limit of no mean speed $v_{\mu}$ we expect a relaxation to a maxwellian velocity distribution. This can be seen by converting back to Cartesian velocity components.

$$
\lim _{v_{\mu} \rightarrow 0} e^{-\left(\frac{\sqrt{v_{x}^{2}+v_{y}^{2}+v_{z}^{2}}-v_{\mu}}{\sigma}\right)^{2}}=e^{-\frac{v_{x}^{2}+v_{y}^{2}+v_{z}^{2}}{\sigma^{2}}}=f_{m}
$$

Second, the distribution approaches an infinitesimally thin velocity surface as the width of the thermal component approaches zero, $\sigma \rightarrow 0$. This describes an isotropic monoenergetic distribution.

$$
\lim _{\sigma \rightarrow 0} e^{-\left(\frac{\sqrt{v_{x}^{2}+v_{y}^{2}+v_{z}^{2}}-v_{\mu}}{\sigma}\right)^{2}}= \begin{cases}1, & \text { if } \sqrt{v_{x}^{2}+v_{y}^{2}+v_{z}^{2}}=v_{\mu}^{2} \\ 0, & \text { if } \sqrt{v_{x}^{2}+v_{y}^{2}+v_{z}^{2}} \neq v_{\mu}^{2}\end{cases}
$$

The equation for the probe current is expressed in terms of the velocity components in the $2 \mathrm{D}$ plane intercepting the probe, and hence the distribution becomes

$$
f\left(v_{x}, v_{y}\right)=\int_{-\infty}^{\infty} e^{-\left(\frac{\sqrt{v_{x}^{2}+v_{y}^{2}+v_{z}^{2}}-v_{\mu}}{\sigma}\right)^{2}} d v_{z} .
$$

A slice of $f\left(v_{x}, v_{y}\right)$ is shown in Fig. 6 revealing its dependence on $v_{\mu}$ and illustrating its convergence on the monoenergetic isotropic distribution in the limit as $\sigma \rightarrow 0$. 
Further support for the use of the proposed isotropic mean energy distribution $f_{\mu}$ has been given by 2D PIC code simulations using the commercially available OOPIC code ${ }^{28,29}$. The simulated dimensions and parameters were designed to approximately reproduce the experimental conditions explored in this paper. The simulation geometry was in a $2 \mathrm{D}$ slice through the centre of the device, intersecting four of the six Polywell coils. The simulated Polywell had a coil current of 7950 Amp turns, giving a peak field in the coil face of $B_{\text {peak }}=$ 0.14 T. Four simulated electron sources surrounded the coils, each emitting a current of $0.2 \mathrm{~mA} / \mathrm{cm}$ (note this is expressed as a current per unit of height because the simulation is $2 \mathrm{D}$ ). The extraction voltage was set to $V_{\text {bias }}=130 \mathrm{~V}$, and the resulting potential well formed by the electrons is $35 \mathrm{~V}$ deep. The velocity components of the simulated particles have been extracted at four spatial positions along the coil axis, extending from the centre of the null to the field coil. The extracted velocity data has been used to create a plot of the projected 2D velocity distribution function, $f\left(v_{x}, v_{y}\right)$. A 1D slice of that function is plotted in Fig. 7 and shows reasonable agreement with our proposed function, plotted in Fig. 6 . Further discussion and analysis of the simulation results are left for further work, and is only presented here to support the proposed distribution function.

Substituting Eqn. 11 into Eqn. 3 gives the $I(V)$ characteristic for the isotropic mean energy distribution, $I_{\mu}(V)$. Two data sets are compared with their respective fits in Figure 8. In both cases the data has been collected from a single Langmuir probe located in the centre of the device. The Polywell bias voltage was held constant at $V_{\text {Bias }} \simeq 112 \mathrm{~V}$, extracting a current of $I_{\text {Beam }} \simeq 2.7 \mathrm{~mA}$. The coil current $I_{\text {Poly }}$ was varied to contrast the $B_{\text {peak }}=0$ $\mathrm{T}$ case (no field in the coil face) with $B_{\text {peak }}=15 \mathrm{mT}$. At electron energies in the range of $20 \mathrm{eV}$ to $100 \mathrm{eV}$, a magnetic field of $15 \mathrm{mT}$ is sufficient to force the electron gyroradius to approach $10 \%$ of the device radius, $r_{g}<10 \% R_{D}$, which is the approximate condition for reflection from the adiabatic flux surface at the magnetic null boundary ${ }^{17}$. Hence $B$ is sufficiently large to prolong the electron confinement in the core.

Note that an equally good qualitative fit to the data can be made with the drifting/beam maxwellian distribution, giving only negligible difference in the fit residuals (not plotted for clarity). Both distributions can be made to fit the data but give different plasma parameters in Table I. The reason for this similarity in the underlying $I(V)$ functions is that Langmuir probes only measure relative energy, not potential. As a result, a Langmuir probe can be considered as a particle energy filter. Although it can be shown that a Maxwellian fit is not 
appropriate to either of these data sets, it might be impossible to conclusively prove which distribution is correct without resorting to an extra diagnostic that can provide additional information $^{25}$. However, due to the magnitude of the applied magnetic field, ballistic reflections are likely to occur and will effectively make the beam distribution isotropic, and hence Eqn. 11 should be the most appropriate distribution of the two discussed. The results of the OOPIC simulation in Fig. 7 support this hypothesis.

It is worth noting that regardless of which of the two distributions is applied, the plasma potential $V_{p}$ has decreased relative to the $B=0$ case. This is consistent with an increase in potential well depth, relative to the potential on the coils, due to an increase in the dwell time of the electrons in the presence of a magnetic field. However, the measured electron densities for the two cases were not significantly different, which would appear to oppose a claim of potential well formation. A possible explanation for this is that the application of the magnetic field causes the unidirectional beam to spread out isotropically to a larger volume within the device, which would cause a decrease in density. However, an increase in the electron dwell time within the device will lead to a higher electron recirculating current, thus increasing the density. Although the increase in this density is similar or equal to the density of the initial electron beam, a deeper potential well will result since the electrons occupy a larger volume.

If a potential well has formed then $V_{\mu}$ must drop to conserve energy as the electrons transit into the well centre. However, if the magnetic field has increased the electron confinement time, then the increased electron density superimposes a space charge on to the vacuum potential field such that the electrons may no longer be accelerated to the same energy as in the $B=0$ case. The thermal component, $V_{t h}$, of the energy distribution remains unchanged, which is expected for the low magnetic fields used in this experiment. However, larger magnetic fields should result in an increase in confinement time leading to more thermalisation.

\section{RADIAL PLASMA POTENTIAL PROFILE IN A HIGH MAGNETIC FIELD}

High magnetic fields in the planes of the coils and corner cusps are required to produce sufficient electron confinement in order to establish a virtual cathode in the core of the device. 
It has been shown ${ }^{17}$ there is a non-adiabatic core in the central null region surrounded by adiabatic mirror confinement. Increasing the current in the coils should produce a larger confinement time for electrons within the device. However, this will also result in adiabatic mirror reflections on the outside of the device, thus limiting the injected electron current. Moreover, increasing the coil current further to create large confining fields will result in a reduction in volume of the non-adiabatic core region, which ultimately leads to a smaller confinement volume. When ions are introduced to the device, this might lead to a smaller volume for fusion reactions and limit the device efficiency.

In the experiment reported here, the electron injection parameters and coil currents were kept constant so that the effects of varying electron confinement time, volume and admittance do not produce additional confounding effects to the probe results. The probe located at the centre of the device, Probe A, was mounted on a translation stage that could move radially in increments of $3 \mathrm{~mm}$. At each spatial point the experiment was repeated under the same initial conditions, allowing a measurement of the spatial change in the $I(V)$ characteristic across the device radius. All probe traces were analysed with the isotropic mean energy distribution. Some sample probe traces at four different spatial points are shown in Figure 9.

In the discussion of the previous section, the isotropic mean energy characteristic, $I_{\mu}(V)$, was shown to accurately represent the underlying EEDF in the null region. As the probe moves into higher $B$ field regions, we expect to see a growing disparity between the predicted $I(V)$ and the measured data in the attractive saturation current region. This is because the magnetic field limits electron transport across magnetic field lines to the probe, and hence limits the attainable saturation current. The result is an asymmetric sheath around the probe which significantly complicates the $I(V)$ characteristic calculation.

This deviation becomes progressively larger as the B field increases and the electron gyroradius $r_{g}$ approaches the probe radius. The parameters in this experiment have been chosen to minimise this effect over a large spatial range of the device. For the radial positions ranging from $0 \mathrm{~cm}$ to $2 \mathrm{~cm}$, the gyroradius is expected to be at least ten times larger than the probe radius, $r_{g} \geq 10 r_{p}$, and approaches $3 r_{p}$ in the limit of the maximum magnetic field in the coil face. This means that over a wide range of measurement points, the gyroradius $r_{g} \gtrsim \lambda_{d}$ and hence the deviation from the prediction is expected to be minimal. A sample of the typical deviation in the saturation region is shown in Figure 10. The deviation in 
the saturation region was excluded from the curve fitting procedure. In figure 10 the fitting algorithm was restricted to the data range of $-10 \mathrm{~V}$ to $+60 \mathrm{~V}$.

Although we would expect the magnetic field to alter the distribution function measured with the probe, we anticipate that when $r_{g}>r_{p}$ the magnetic field acts to locally randomise the incoming electron trajectories at the probe sheath, and acts to maintain the mean energy isotropic distribution observed in the magnetic null. This hypothesis is supported by the OOPIC case study presented in Fig. 7, where the velocity distribution within the point cusp is similar to the predicted distribution presented in Fig. 6. Hence, we have used the mean energy isotropic $I_{\mu}(V)$ for data fitting for magnetic fields that range from the magnetic null at the device centre to the maximum field, $B_{\text {peak }}$, in the plane of the coils. It was assumed that any fitting errors become progressively larger as $B_{\text {peak }}$ was approached.

The resulting data for the radial plasma potential profile is shown in Fig. 11. The error bars were calculated from the error in the fit residuals during the fitting process. Note that there is a potential well (also known as a virtual cathode ${ }^{4,5}$ ) of $-10 \mathrm{~V}$ with a $2 \mathrm{~cm}$ radius from the centre. The fitting procedure is accurate over this region since the magnetic field is relatively low and the effects of the magnetic field on the distribution function are well characterised. However, there is more uncertainty about the values of the plasma potential in the $2-3.5 \mathrm{~cm}$ region, due to the higher magnetic fields, and are likely to represent an underestimate of the potentials.

The potential well formed can be compared with an analytical estimate from Poisson's equation if we assume that the well is approximately spherically symmetric and the electron density is approximately constant as a function of radius. In spherical coordinates Poisson's equation is

$$
\frac{1}{r^{2}} \frac{\partial}{\partial r}\left(r^{2} \frac{\partial}{\partial r} V\right)=-\frac{n_{e} q}{\epsilon_{0}} .
$$

Under the stated conditions we set the boundary condition $\partial V / \partial r=0$ at $r=0$. Moreover, we set $V=0$ at radius $r=R$. Using both of these boundary conditions equation 12 can be solved to yield the relationship

$$
V_{w e l l}=-\frac{n_{e} q R^{2}}{6 \epsilon_{0}} .
$$

Using the approximate density $n_{e}=10^{8} \mathrm{~cm}^{-3}$ and radius $R=0.01 \mathrm{~m}$, we obtain $V_{\text {well }}=$ 
$-30 \mathrm{~V}$. This deviation from the measured value is a result of the simplified assumptions stated above. A more accurate calculation of the potential well will need to take into account the real spatial density profile of the electrons.

\section{SCALING WITH B AND E}

To allow the measurement of the potential well variation with changes in magnetic field $B$, electric field bias $V_{\text {bias }}$, and electron density $n_{e}$, two probes were used to simultaneously measure $V_{p}$ at different radial points. Probe A was mounted at the centre of the device, and the other, Probe B, at a radial position of $1.8 \mathrm{~cm}$, which was approximately halfway along the device radius. In the first experiment, the magnetic field was varied such that the peak magnetic field in the coil face varies from $B_{\text {peak }}=0$ to $B_{\text {peak }}=26 \mathrm{mT}$. The measured plasma potential from the two probes as a function of the maximum magnetic field is given in Fig. 12. When the magnetic field is relatively weak, the potential difference between the probes indicates that no potential well is present, because the $V_{p}$ at the centre probe is more positive than at Probe B. When the magnetic field reaches some crucial minimum value, 5.5 $\mathrm{mT}$ in this experiment, the potential difference between the two probes is inverted and a potential well begins to form and is clearly measurable. Moreover, the potential difference between the two probes becomes progressively larger with increasing magnetic field.

There are significant challenges in designing an experiment that can be used to accurately characterise the way the potential well scales with B because the size of the adiabatic flux surface becomes progressively smaller with increasing B. Hence, the position of Probe B would have to be adjusted for each data point to compensate for the change in well size. If we can assume that the difference between the two probes is indicative of the overall change in the potential well depth we can obtain a relationship for the scaling of potential well with $\mathrm{B}$. The analysis has been restricted to data in the domain of $>15 \mathrm{mT}$ because it is unclear if a potential well has formed for fields at lesser magnitudes. A plot of the potential difference between the two probes is shown in Fig. 13 and scales approximately linearly with magnetic field $B$. However, a greater measurement range would be needed to confirm this relationship, and also a more detailed study is required to determine how best to characterise the change in well depth with $B$.

In another experiment the magnetic field was held constant at $B_{\max } \simeq 0.16 \mathrm{~T}$ while 
the Polywell bias voltage was varied from $V_{\text {bias }}=93 \mathrm{~V}$ to $122 \mathrm{~V}$, the data is shown in Fig. 14. Although changing the bias voltage effectively changes the injection energy of the electrons, it also changes the injection current, and hence affects the ultimate electron density $n_{e}$ obtained in the well. It was not possible to separate these two effects because they have an opposite effect on the results. An increase in the electron density, with increasing injection current, leads to a linear increase in the potential well depth, $V_{w e l l}$, given in Eqn. 13. Increasing the extraction voltage gives an exponential increase in the current emitted from the filaments, but does not necessarily mean an exponential increase in the current entering the Polywell since this is a complex function of the geometry and is space charge limited.

By comparison, increasing the energy of the electrons leads to a decrease in the electron confinement time because a higher energy electron population needs a higher magnetic field to achieve the same degree of adiabatic mirror confinement. However, this effect is at a maximum when the adiabatic flux surface becomes larger than half the device radius, beyond which the degree of mirror confinement decays rapidly. Fig. 15 supports this hypothesis since it shows the potential well scales linearly with injected current until the injected electron energy is sufficiently large such that competing effects are introduced.

\section{CONCLUSIONS}

Biased Langmuir probes have been used to characterise potential well formation as a function of a number of Polywell parameters, such as magnetic field strength and injection energy. Orbital Limited Motion theory was used to measure and confirm the EEDF of injected electrons. This information was combined with knowledge of the types of possible electron trajectories ${ }^{15,17}$ to propose an EEDF for the electron population in the magnetic null. The proposed EEDF $I_{\mu}(V)$ is in good agreement with the collected data.

The proposed $I_{\mu}(V)$ was used to study the spatial change of the plasma potential $V_{p}$ across the device, and confirmed a potential well had formed. However the shape of the potential well did not agree with the simple parabolic shape predicted by Poisson's equation and previously measured by Krall ${ }^{13}$. Potential well formation was found to scale linearly with increasing magnetic field and injection current, in good agreement with the currently proposed confinement models. Dependence on injection energy was also measured, but 
obscured by the competing effect of injection current scaling.

The proposed $I_{\mu}(V)$ provides a powerful diagnostic for studying Polywell physics providing $f_{\mu}\left(v_{r}\right)$ is an accurate representation of the underlying EEDF. To confirm the interpretation of the $I(V)$ is correct, the biased probe technique discussed in this paper should be combined with another probe technique that is less dependant on the shape of the EEDF. If the $V_{p}$ 's of the two techniques are in agreement, then all other derived parameters from $I_{\mu}(V)$ can be considered accurate. One such technique is the capacitive probe technique used previously on the HEPS experiment ${ }^{13}$. Additionally, emissive probes have been shown to allow uncomplicated analysis of the $V_{p}$ through the deviation potential in electron only plasmas $^{30,31}$.

\section{ACKNOWLEDGMENTS}

M.C. wishes to acknowledge A. Samarian for productive discussions on Langmuir Probe diagnostics. M.C. was supported by a UPA postgraduate scholarship from the University of Sydney. This work was performed in partial fulfilment of the degree requirements for a Ph.D.

\section{REFERENCES}

${ }^{1}$ P. T. Farnsworth, U.S. Patent 3,258,402 (1966).

${ }^{2}$ P. Farnsworth, US Patent 3,386,883 (1968).

${ }^{3}$ R. Hirsch, J. Appl. Phys. 38, 4522 (1967).

${ }^{4}$ R. Hirsch, Phys. Fluids 11, 2486 (1968).

${ }^{5}$ W. Elmore, J. Tuck, and K. Watson, Phys. Fluids 2, 239 (1959).

${ }^{6}$ O. A. Lavrentev, Ukr. Fiz. Zh. 8, 440 (1963).

${ }^{7}$ R. L. Hirsch, Phys. Fluids 11, 2486 (1968).

${ }^{8}$ G. H. Miley, J. Nadler, T. Hochberg, Y. Gu, O. Barnouin, and J. Lovberg, Fusion Technol. 19, 840 (1991).

${ }^{9}$ J. Berkowitz et al., Proceedings of the 2nd Int. Conference on Peaceful Uses of Atomic Energy. 1, pages 146-155 (1958).

${ }^{10}$ H. Grad, Phys. Rev. Lett. 4, 222 (1960). 
${ }^{11}$ R. Bussard, Fusion Technol. 19, 273 (1991).

${ }^{12}$ N. Krall, Fusion Technol. 22, (1992)

${ }^{13}$ N. Krall, M. Coleman, K. Maffei, J. Lovberg, R. Jacobsen, and R. Bussard, Phys. Plasmas 2, 146 (1995).

${ }^{14} \mathrm{R}$. Bussard, in 57th International Astronautical Congress, IAC (2006).

${ }^{15}$ See National Technical Information Service Document No. ADA257648. (R. Bussard and N. Krall, EMC2 Technical Report 0191-02, Feb 1991). Copies may be ordered from the National Technical Information Service, Alexandria, VA 22312.

${ }^{16}$ T. Dolan, Plasma Phys. Controlled Fusion 36, 1539 (1994).

${ }^{17}$ M. Carr, D. Gummersall, S. Cornish and J. Khachan, Phys. Plasmas 18, 112501 (2011).

${ }^{18}$ M. Carr and J. Khachan, Phys. Plasmas 17, 052510 (2010).

${ }^{19} \mathrm{~F}$. Chen, Introduction to plasma physics and controlled fusion, Vol. 1 (Plenum press, 1984).

${ }^{20}$ M. Rosenberg, and N. Krall, Phys. Fluids B 4, 1788 (1992).

${ }^{21}$ F. Magnus and J. T. Gudmundsson, Rev. Sci. Instrum. 79, 073503 (2008).

${ }^{22}$ A. Savitzky and M. J. E. Golay, Anal. Chem. 36, 1627 (1964).

${ }^{23}$ H. M. Mott-Smith and I. Langmuir, Phys. Rev. 28, 727 (1926).

${ }^{24}$ A. H. Heatley, Phys. Rev. 52, 235 (1937).

${ }^{25}$ N. Hershkowitz, in Plasma Diagnostics, edited by O. Auciello, D. L. Flamm (Boston, Academic Press, 1989), p. 113-183.

${ }^{26}$ I.B. Bernstein and I.N. Rabinowitz, Phys. Fluids 2, 112 (1959).

${ }^{27}$ B. Polychronopulos, Plasma Phys. 15, 37 (1973).

${ }^{28}$ J.P. Verboncoeur, in Plasma Science, 1995. IEEE Conference Record-Abstracts., 244 (1995).

${ }^{29}$ D.L. Bruhwiler et. al., Phys. Rev. ST Accel. Beams 4, 101302 (2001).

${ }^{30}$ J.P. Sheehan and N. Hershkowitz, Plasma Sources Sci. Technol. 20, 063001 (2011).

${ }^{31}$ J.P. Kremer et. al., Rev. Sci. Instrum. 78, 013503 (2007).

${ }^{32}$ J. Berkowitz, H. Grad, and H. Rubin, Proceedings of the 2nd Int. Conference on Peaceful Uses of Atomic Energy. 1, pages 177-189, (1958). 
FIGURES 


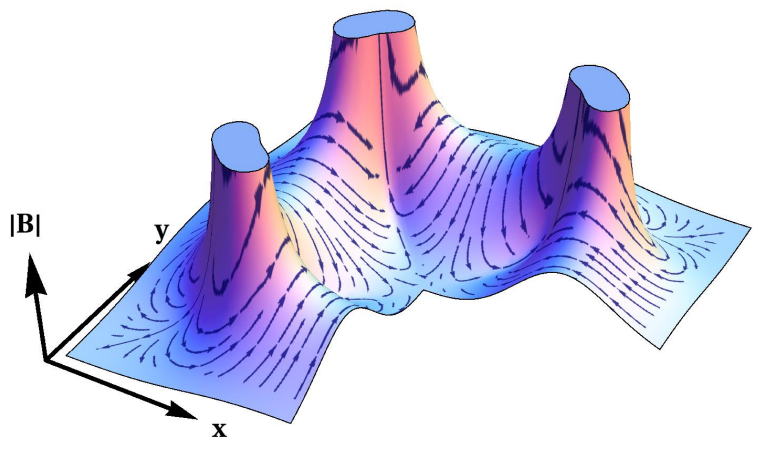

FIG. 1. The magnitude of the magnetic field B plotted in the $x-y$ plane intercepting four of the six field coils. The fieldlines are superimposed over the field's magnitude plot to reveal the underlying magnetic cusp structure of the Polywell ${ }^{12,17}$. 


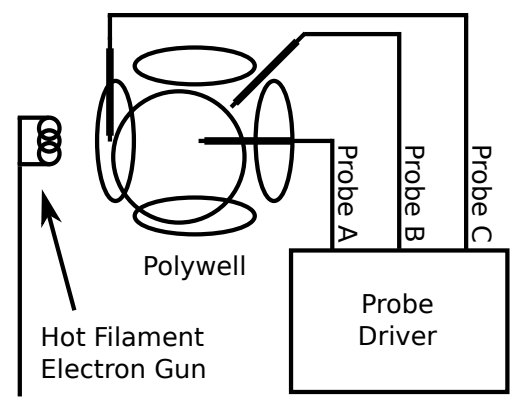

FIG. 2. A diagram of the experimental setup showing the key biased probe positions. 


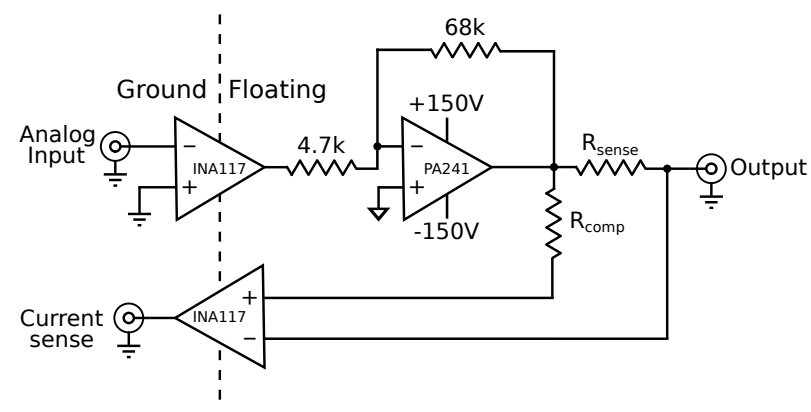

FIG. 3. A high level diagram of the biased Langmuir probe driver circuit reduced to its simplest underlying components. 


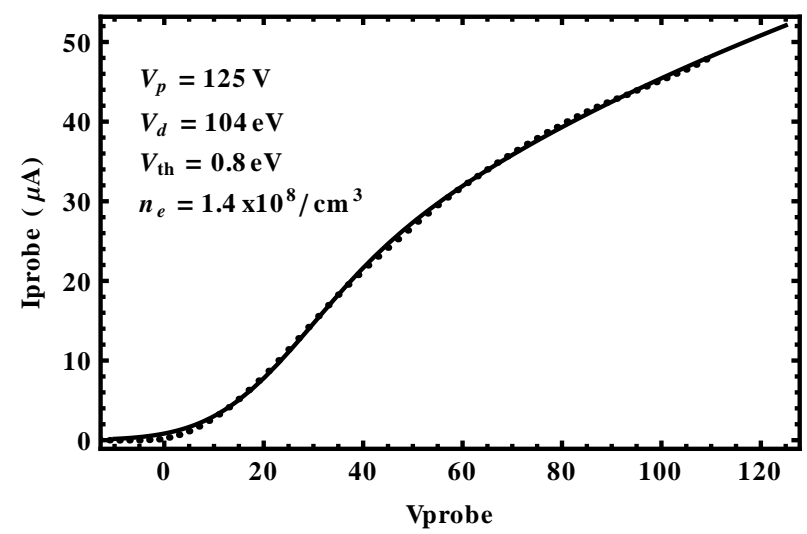

FIG. 4. A sample $I(V)$ curve fitted with the predicted function for a drifting-maxwellian. In this experiment the probe was placed in the centre of the coil nearest to the single filament being tested. No magnetic field was present for the test. The Polywell was biased to a voltage of $150 \mathrm{~V}$ drawing a current of $4.5 \mathrm{~mA}$. 


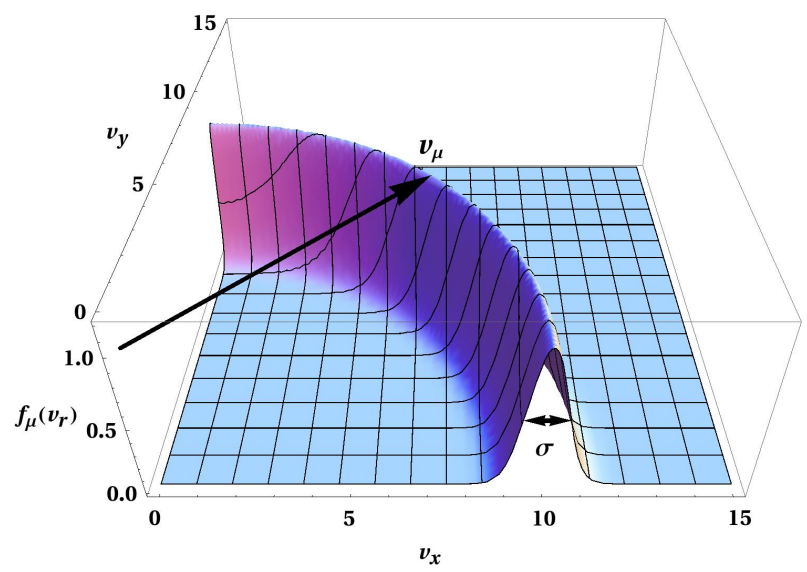

FIG. 5. A plot of the mean energy isotropic distribution $f_{\mu}\left(v_{r}\right)$ in one quadrant of a 2D Cartesian plane. When $\sigma \rightarrow 0$ this plot becomes a slice through a sphere. In this plot, the function $f_{\mu}\left(v_{r}\right)$ has not yet been normalised, such that integration over all $\mathbf{v}$ yields 1 . Note that this plot is a slice in a Cartesian plane, and distinctly different from the projection of $f_{\mu}\left(v_{r}\right)$ in to the 2D plane at right angles to the probe, which is plotted in Fig. 6. 


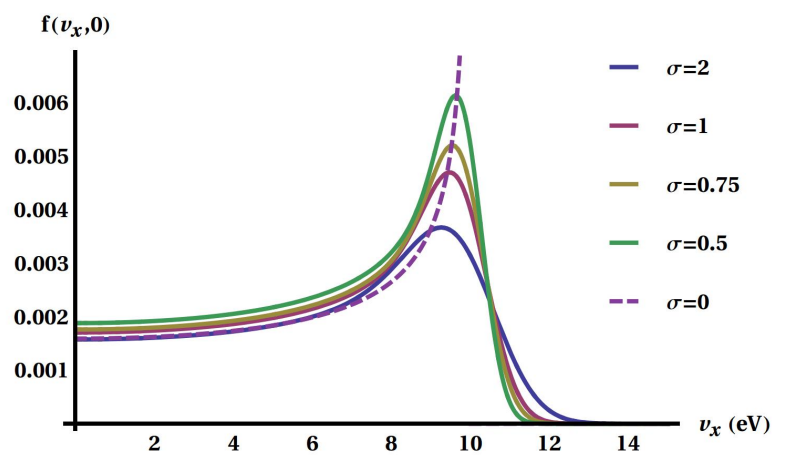

FIG. 6. Convergence of Eqn. $11 f\left(v_{x}, 0\right)$ on the monoenergetic isotropic distribution. The case shown has $v_{\mu}=10 \mathrm{eV}$. As $\sigma \rightarrow 0$ the result converges on $1 / \cos \phi$ which is the analytical result for the monoenergetic isotropic case ${ }^{23}$. 




FIG. 7. A plot of the velocity distribution $f\left(v_{x}, 0\right)$ from simulated data. The commercial OOPIC code was used to simulate an electron plasma with conditions approximating our experiment. The particle data was sampled in a number of spatial locations along the coil face axis. This plot shows reasonable agreement with the predicted function shown in Fig. 6 and thus supports the theoretically proposed $I_{\mu}(V)$. 


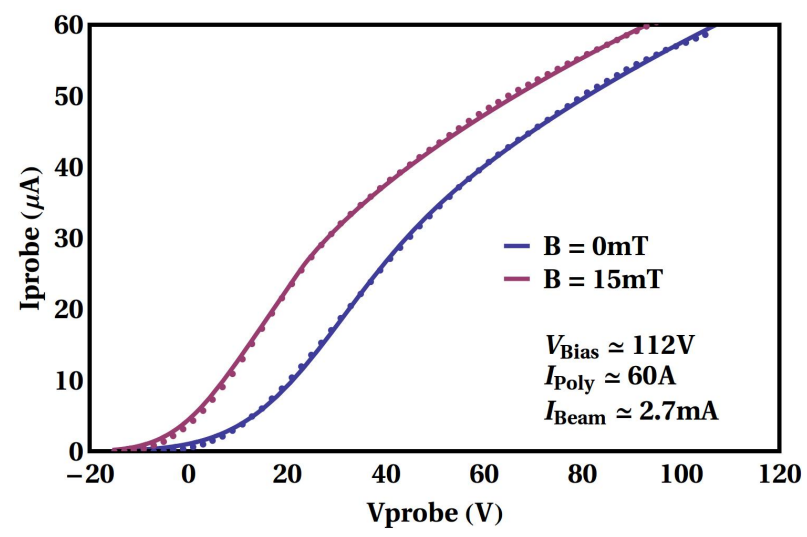

FIG. 8. The two magnetic field test cases, both measured on Probe A in the centre of the device. The fitted parameters for the two data sets with their respective $I(V)$ characteristic models are given in Table I. 


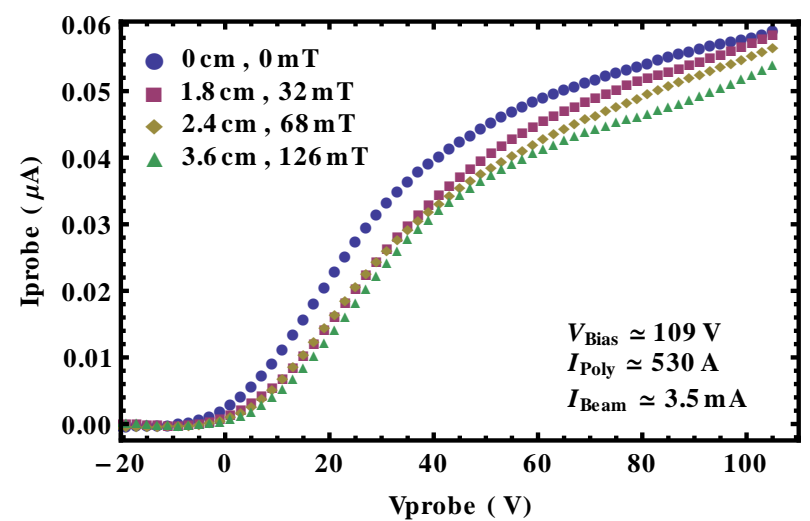

FIG. 9. Four example $I(V)$ datasets from the high magnetic field radial profile experiment. Each dataset has been taken at a different spatial location along the coil axis. 


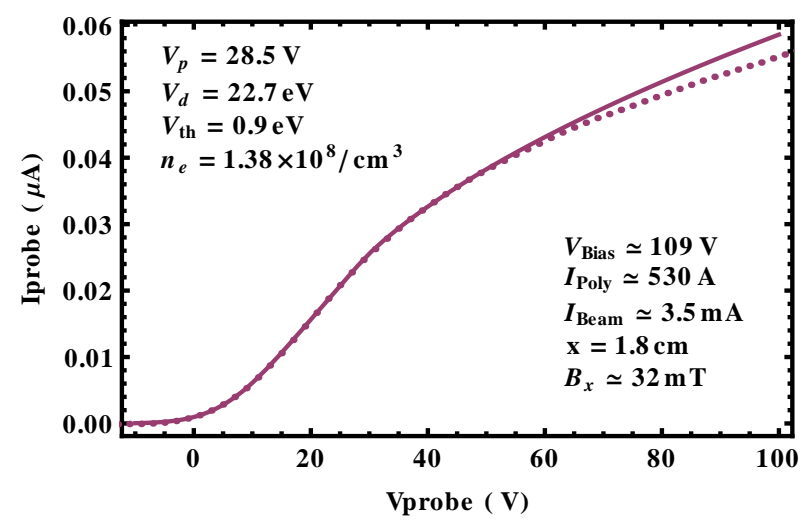

FIG. 10. Sample of the fitting in a strong magnetic field. Shows the deviation from the fitted $I(V)$ in the saturation region. 


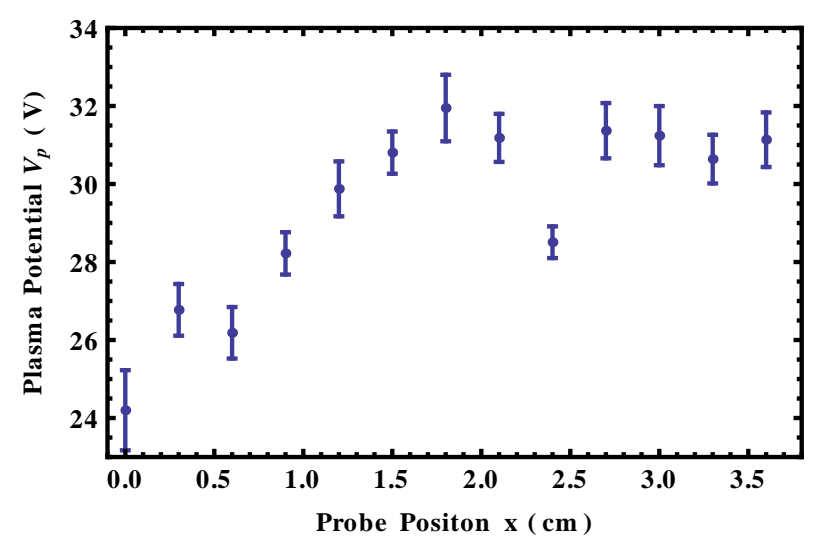

FIG. 11. The spatial profile of the plasma potential in a strong magnetic field. The $I(V)$ characteristic at each spatial point has been fitted with the $I_{\mu}(V)$ to find the $V_{p}$ spatial profile. This data shows a potential well has formed in the middle of the device. 


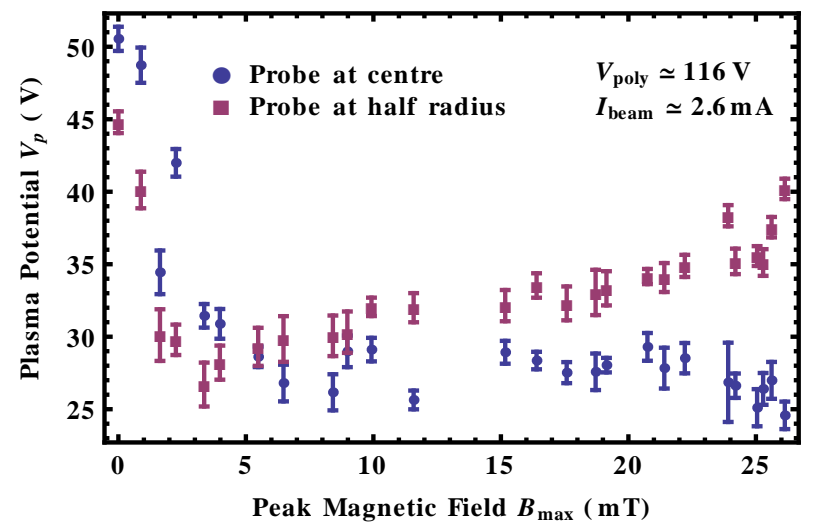

FIG. 12. Potential well formation in the low magnetic field range. The plasma potential $V_{p}$ for Probes A and B is shown over a range of relatively low peak magnetic field values. As the field increases, the potential difference between the probes eventually becomes inverted and becomes progressively larger with increasing magnetic field strength. 


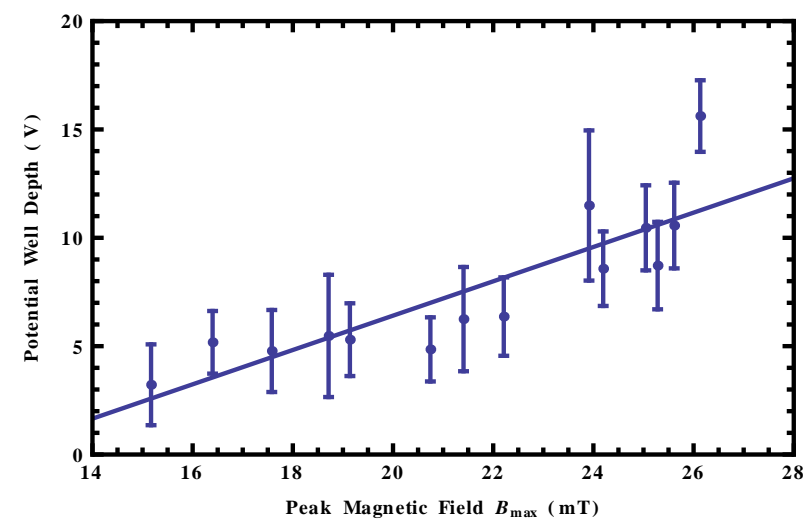

FIG. 13. Potential well scaling with B. For each data point in Fig. 12 with $B_{\max }>15 \mathrm{mT}$ the difference in the two probe potentials is taken to be indicative of the change in overall potential well depth. The resulting trend is approximately linear. 


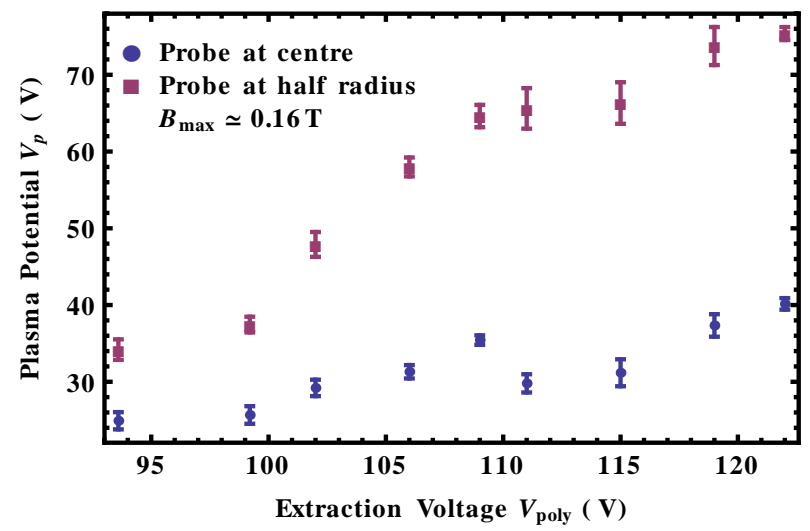

FIG. 14. The change in potential well formation with extraction voltage, $V_{\text {poly }}$. The variation is determined by two competing parameters, the injected electron density and average energy. 


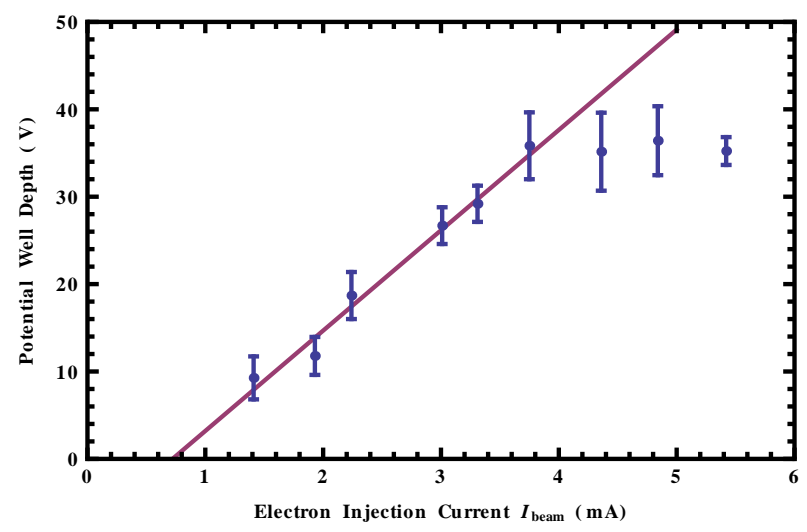

FIG. 15. Potential well scaling with the injected electron current $I_{\text {beam }}$. Here the potential difference between the two probes in Fig. 14 is assumed to be indicative of the overall change in well depth, and plotted as a function of the injected beam current. 
TABLES 


\begin{tabular}{lccc}
\hline \hline Dataset & \multirow{2}{*}{$B=0$} & \multicolumn{2}{c}{$B=15 \mathrm{mT}$} \\
Model & Beam & Iso. & Beam \\
\hline$V_{p}$ & $87.2 \mathrm{~V}$ & $22.9 \mathrm{~V}$ & $56.1 \mathrm{~V}$ \\
$V_{d}, V_{\mu}$ & $65.5 \mathrm{eV}$ & $23.7 \mathrm{eV}$ & $49.7 \mathrm{eV}$ \\
$V_{t h}$ & $1.3 \mathrm{eV}$ & $1.1 \mathrm{eV}$ & $0.72 \mathrm{eV}$ \\
$n_{e}$ & $1.7 \times 10^{8} \mathrm{~cm}^{-3}$ & $1.4 \times 10^{8} \mathrm{~cm}^{-3}$ & $1.7 \times 10^{8} \mathrm{~cm}^{-3}$ \\
\hline \hline
\end{tabular}

TABLE I. Fitted parameters for the two data sets shown in Figure 8. For the case of $B=15 \mathrm{mT}$, fits to both models have been listed. 